\title{
A Tool for Setting Therapeutic Goals by the Multidisciplinary Team for the Preschool Child With ASD (Autism Spectrum Disorder)
}

\author{
Kotsopoulou Angelique, Georgiou Anastasia, Gyftogianni Maria, Gyftogianni Kataerina, \\ Sakellari Marigo, Troupou Antigoni, Florou Irene \\ Day Centre for Children with Developmental Disorders, Messolonghi, Greece
}

\begin{abstract}
The clinical profile of children with ASD (autism spectrum disorder) varies at the early stages of development. A variety of specific deficits may be observed, e.g., feeding difficulties, motor and verbal dyspraxia, sensory integration deficits, etc., therefore the need for multidisciplinary observation and assessment is necessary for setting realistic therapeutic goals. In 2007, the interdisciplinary team of the Day Centre developed a behavior observation tool (EDALFA-A tool of the Interdisciplinary Team for the evaluation of the level of functioning of the child with ASD) which provides a clinical profile of the child's level of function and detailed targets for intervention. EDALFA consists of a developmental scale based on eight international scales and on systematic review of the recent literature. It includes the following observation measures: motor development cognitive development, speech and language development psychosocial development, everyday skills, play, other (joined attention, imitation, stereotypes). In every one of those functions, at each age level (1 month to 6 years) skills ranging from 0 to 9 are described, which the typically developing child is expected to master. Upon admission to the program, three therapists (psychologist, speech, occupational) observe systematically the child on a sufficient number of sessions and jointly complete the EDALFA protocol, which shows the developmental profile of the child compared to the typically developing child and the goals of therapy in a hierarchical way. To assess reliability of the tool, a study was carried out that compared the performance of 30 children ( 2 yrs to 5 yrs 11 mon) on the EDALFA and on Vineland questionnaires filled by the parents. Highly significant correlations were observed on the common measures of the two tools.
\end{abstract}

Keywords: Autism, multidisciplinary team, developmental scales, dyspraxia, sensory integration, feeding difficulties

\footnotetext{
*Acknowledgements: The authors acknowledge the contribution of Ms. E. Koymanioti for the statistical work of the study. Kotsopoulou Angelique, Ph.D., speech language pathologist, associate professor, Day Centre for Children with Developmental Disorders.

Georgiou Anastasia, social worker, Day Centre for Children with Developmental Disorders.

Gyftogianni Maria, speech language therapist, Day Centre for Children with Developmental Disorders. Gyftogianni Kataerina, speech language therapist, Day Centre for Children with Developmental Disorders. Sakellari Marigo, teacher, Day Centre for Children with Developmental Disorders.

Troupou Antigoni, speech language therapist, Day Centre for Children with Developmental Disorders. Florou Irene, Msc., psychologist, Day Centre for Children with Developmental Disorders.
} 


\section{Introduction}

Recent studies have shown that the diagnosis of autism can reliably be made by the 24th month of age (Ozonoff, Iosif, Baguio, \& Hepburn, 2010; Georgiades, Szatmari, Zwaigenbaum, Bryson, \& Brian, 2013) and that early intervention improves substantially the prognosis (Dawson et al., 2010; Helt at al., 2008). The clinical profile of children with ASD (autism spectrum disorder) varies considerably at these early stages of development. Besides the cardinal signs of autism, that is impairment in social communication, stereotypes and linguistic delay, a variety of specific deficits may be observed, e.g., difficulties in joined attention (Watson, Baranek, Crais, Reznick, Dykstra, \& Perryman, 2007; Sullivan, Finelli, Marvin, Garrett-Mayer, Bauman, \& Landa, 2007), feeding difficulties (Arvedson \& Brodsky, 2002; Schreck, Williams, \& Smith, 2004; Emond, Emmett, Steer, \& Golding, 2010), sensory integration deficits (Ayres, 2005; Scott \& Winnie, 2007) motor and verbal dyspraxia (Dziuk, Gidley, Apostu, Mahone, Denkia, \& Mostofski, 2007; Tierney, Kurtz, \& Souders, 2012), therefore, it is necessary for multidisciplinary observation and assessment for setting realistic therapeutic goals.

Until recently, most of the assessment tools of the level of functioning of children with ASD relied on parent reports (Vineland, Cars) or on short observations by one or two therapists usually psychologists or psychiatrists. Taking into consideration that a considerable number of children at this early age present with specific problems as those mentioned above, the need for multidisciplinary observation and assessment is necessary for placement of realistic therapeutic goals. In 2007, the interdisciplinary team of a Day Centre in Greece developed a tool of the multidisciplinary team for the assessment of the functional level of children with ASD-EDALFA (Kotsopoulos et al., 2010) which provides a clinical profile of the child's level of function and detailed goals for the intervention.

\section{Method Materials}

\section{Description of the Tool}

EDALFA is not a test for the diagnosis of autism; it is a tool for the multidisciplinary team to assess the child on important psycholinguistic and motor functions, which would allow them to set joint realistic treatment aims and goals. It consists of: (1) manual with detailed descriptions of the tool's usage; (2) answering forms or protocols; and (3) a scale of the levels of typically developing young children in various functions, with special emphasis in the first two years of life and on skills that are not usually included in other developmental scales.

The description of the stages of normal development is provided for every month for the first year of life, every two months until the age of two, and every six till the age of six. The developmental levels of the child with autism are compared with those of typically developing children in order to determine his/her profile, which would direct the treatment team to set realistic therapeutic goals for the intervention.

The developmental scale was based on five international scales (Zimmerman, Steiner, \& Pond, 1992; Hindley, 1960; Bzoch \& League, 2000; Mecham, 1958; Arverson, 2007; Fewell \& Folio, 2000) and systematic review of the recent literature for inclusion of skills identified as important in the development of the child, e.g., joined attention, imitation, stereotypies etc.. The developmental scale includes detailed information on the development of the following functions: motor development (gross and fine movements), cognitive 
development, speech and language development (comprehension and expression), psychosocial development (emotions-social skills), everyday skills (feeding, dressing, toilet training), play, and other (joined attention, imitation, stereotypes).

For each one of those functions, at each age level (one month to six years), there are from 0 to 9 skills that the typically developing child is supposed to have mastered. Table 1 presents an example of a table of the skills that a toddler 12 to 14 months has usually mastered.

In the answering forms of the tool, there are all the age levels from one month to six years and next to each one of them the number of skills that the typically developing child has mastered (see Table 2).

Table 1

An Example of Typical Development: The Child 12-14 Months of Age

\begin{tabular}{|c|c|c|c|c|c|c|c|c|}
\hline \multicolumn{9}{|l|}{ Typical Development } \\
\hline \multicolumn{9}{|l|}{ Age: $12-14$ months } \\
\hline \multicolumn{2}{|c|}{ Motor } & \multirow[t]{2}{*}{ Cognitive } & \multicolumn{2}{|c|}{ Language } & \multirow[t]{2}{*}{ Psycho social } & \begin{tabular}{|l|} 
Skills of \\
everyday life
\end{tabular} & Play & Other \\
\hline Gross & Fine & & Receptive & Expressive & & $\begin{array}{l}\text { Feeding, } \\
\text { dressing, toilet } \\
\text { training }\end{array}$ & & \begin{tabular}{|l|} 
Imitation \\
joined \\
attention \\
stereotypes \\
\end{tabular} \\
\hline $\begin{array}{l}\text { 1. Walks five steps } \\
\text { without support. } \\
\text { 2. Climbs steps } \\
\text { crawling. } \\
\text { 3. Holding a toy can } \\
\text { make } 3 \text { steps } \\
\text { forward. } \\
\text { 4. When on the } \\
\text { knees balances and } \\
\text { turns his head. } \\
\text { 5. Can hold a } \\
\text { medium size ball in } \\
\text { his lap. } \\
6 \text {. Can throw a small } \\
\text { ball to whatever } \\
\text { direction. }\end{array}$ & $\begin{array}{l}\text { 1. Can put top } \\
\text { on a bottle. } \\
\text { 2. Holds two } \\
\text { blocks. } \\
\text { 3. Builds tower } \\
\text { with three. } \\
\text { 4. Can turn a } \\
\text { few pages. }\end{array}$ & $\begin{array}{l}\text { 1. Recognizes and } \\
\text { shows one to three } \\
\text { real objects. } \\
\text { 2. Recognizes } \\
\text { parts of the body: } \\
\text { eyes, nose. } \\
\text { 3. Recognizes } \\
\text { signs of coming or } \\
\text { going: keys, steps, } \\
\text { bell. } \\
\text { 4. Performs simple } \\
\text { acts after showing. } \\
\text { 5. Gives } \\
\text { something when } \\
\text { asked. }\end{array}$ & \begin{tabular}{|l|} 
1. Obeys \\
simple verbal \\
commands. \\
\\
\\
\end{tabular} & $\begin{array}{l}\text { 1. His vocabulary } \\
\text { consists of } \\
\text { approximately } \\
\text { five words. } \\
\text { 2. May say } \\
\text { "gone", "more", } \\
\text { etc. }\end{array}$ & $\begin{array}{l}\text { 1. Seems to } \\
\text { understand the } \\
\text { mood of the } \\
\text { people around } \\
\text { him. } \\
\text { 2. Shows } \\
\text { affection. }\end{array}$ & $\begin{array}{l}\text { 1. Can hold a } \\
\text { cup without } \\
\text { help. } \\
\text { 2. Starts to put } \\
\text { food in his } \\
\text { mouth with a } \\
\text { spoon. } \\
\text { 3. Directs } \\
\text { food in his } \\
\text { moth by hand. }\end{array}$ & $\begin{array}{l}\text { 1. Starts a } \\
\text { simple game, } \\
\text { e.g., pick a } \\
\text { booh by his } \\
\text { own initiative. } \\
\text { 2. Plays with } \\
\text { water and } \\
\text { sand. } \\
\text { 3. Can place } \\
\text { objects in } \\
\text { containers. }\end{array}$ & $\begin{array}{l}\text { 1. Follows the } \\
\text { gaze of an } \\
\text { adult (can } \\
\text { even turn his } \\
\text { head) enen if } \\
\text { there are } \\
\text { obstacles in } \\
\text { the direction } \\
\text { of his gaze. } \\
\text { 2. Imitates } \\
\text { house works. } \\
\text { 3. Rhythmic } \\
\text { stereotypes } \\
\text { continue to be } \\
\text { present. } \\
\text { 4. Can attend } \\
\text { for two } \\
\text { minutes. }\end{array}$ \\
\hline
\end{tabular}

Table 2

Example of a Page of the Answering Form for 12 to 14 Months

\begin{tabular}{|c|c|c|c|c|c|c|}
\hline \multicolumn{7}{|l|}{ Age: $12-14$ months } \\
\hline \multicolumn{7}{|l|}{ Motor } \\
\hline Gross & 1 & 2 & 3 & 4 & 5 & 6 \\
\hline Fine & 1 & 2 & 3 & 4 & & \\
\hline Cognitive & 1 & 2 & 3 & 4 & 5 & \\
\hline \multicolumn{7}{|l|}{ Language } \\
\hline Receptive & 1 & & & & & \\
\hline Expressive & 1 & 2 & & & & \\
\hline Psychosocial & 1 & 2 & & & & \\
\hline Skills of everyday life & 1 & 2 & 3 & & & \\
\hline Play & 1 & 2 & 3 & & & \\
\hline Other (imitation, joined attention, stereotypes) & 1 & 2 & 3 & 4 & & \\
\hline
\end{tabular}




\section{Completing the Answering Form of EDALFA}

The multidisciplinary team at the Day Centre consists of child psychiatrists, speech language therapists, psychologists, occupational therapists, sociologists, social workers, and nursery school teachers. All therapists have received 180 hours of theoretical work and practical work on autism and have experience in the treatment of children with ASD. The diagnosis of autism is done in specialized hospitals with all the necessary neurological and clinical assessments. Furthermore at the Day Centre the Vineland, ADI-R and ADOS are given to every child along by clinical observation of the child by the child psychiatrists. When the diagnosis is completed, the particular case is assigned to three therapists of different disciplines. The therapists in two or alone (according to the severity of autism) have observation sessions with the child. The observation play therapy sessions last from 20 to 30 hour sessions. After the end of the observation period, the therapists involved complete together the Answering form of $\mathrm{E} \triangle \mathrm{A} \Lambda \Phi \mathrm{A}$.

The completion of the Answering form starts from the level of the chronological age of the child and progresses hierarchically to younger ages. For the mastering of a particular skill the symbol $(\mathrm{O})$ is used (circling of the appropriate number). For the skills that are not yet mastered the symbol (X) is used (see Figure $1)$.

\begin{tabular}{|c|}
\hline Age: $12-14$ months \\
\hline Motor: \\
\hline $\begin{array}{llllll}\text { Gross } & 1 & 2 & 3 & 4 & 5\end{array}$ \\
\hline 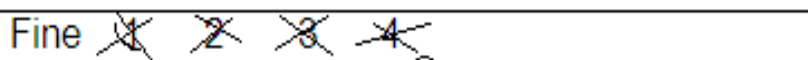 \\
\hline Cognitive: $>$ \& (3) $>$ \& \\
\hline Language: \\
\hline Receptive* \\
\hline Expressive X \\
\hline Psychosocial: 12 \\
\hline $\begin{array}{l}\text { Skills of everyday life: } \\
x \text { (2) (3) }\end{array}$ \\
\hline Play: $\times$ (2) $-8<$ \\
\hline Other: (Imitation, Joined attention, Stereotypes): \\
\hline Commends : \\
\hline
\end{tabular}

Figure 1. Completed answering form.

A function is considered mastered if $60 \%+$ of the corresponding skills have been mastered. In case that they have not, we go back to the immediately previous stage. Upon completion of the answering form the profile of the child is drawn. When the targets of therapy are achieved, a re-evaluation takes place and another $\mathrm{E} \triangle \mathrm{A} \Lambda \Phi \mathrm{A}$ is completed. Below is the profile of a real case George, on first assessment.

\section{History Background}

George was the older boy in a family of two children, sibling's development was typical. Pregnancy, delivery, and first months of development were uneventful. He walked at 13 months. He was assessed at the 
age of two years nine months. At that time he had no speech, limited perception of verbal messages at home. Feeding difficulties: ate under pressure and vomited next. Not responding to his name. Appeared distant and indifferent to parents. Stereotypic, handling of toys watched the examiner but did not respond. Some interest in objects. Handled the telephone and pretended he talked. The diagnosis of autism was made on DSM-IV criteria. George was accepted at the Day Centre and his first EDALFA was completed at 38 months of age, nearly six months after his entrance into the program (see Figure 2).

A summary of the EDALFA assessment at the age of 38 months had as follow: gross and fine movements 36 months, cognitive skills 24 months, speech comprehension 16 months, speech expression of 16 months, psychokinetic skills 22 months, everyday skills 14 months, play 22, and other skills (joined attention, imitation) 22 months.

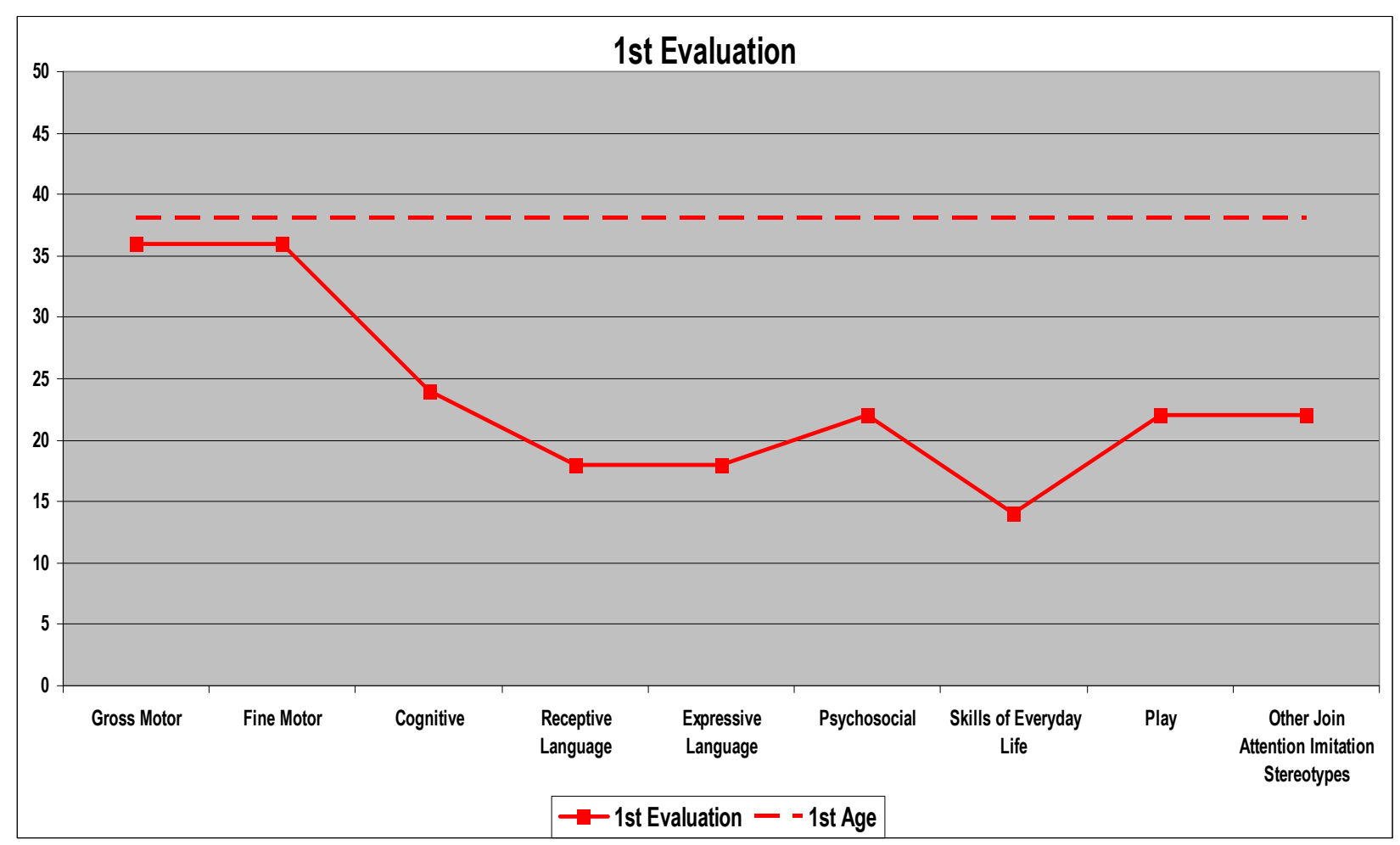

Figure 2. Age of a child in months and his level of functioning on nine measures at the initial evaluation.

As it was mentioned above for every function at each age level there are 1 to 9 skills that the typically developing child has mastered. Upon completion of the $\mathrm{E} \Delta \mathrm{A} \Lambda \Phi \mathrm{A}$ the skills that have not been mastered, by the particular child that has been assessed, are identified and these become the therapy targets. The therapy targets are presented hierarchically from those of earlier stages to those of later.

\section{Therapy Targets (Two of Each Function) for George}

Therapy targets (two of each function) for george are as follows:

(1) Gross motor: (a) to jump with two feet together from the first step of a ladder; and (b) to catch the ball in the air;

(2) Fine motor: (a) to cut paper in the middle; and (b) to copy a circle and a cross;

(3) Cognitive: (a) to start categorizing objects according to shape, color, and function; (b) to point with his 
finger one or two pictures; (c) language Comprehension; (d) to understand words referred to simple space and quantity meanings; (e) to understand simple adjectives, and pronouns;

(4) Expressive language: (a) to use words rather than gestures to express needs; and (b) to name five members of his family;

(5) Psychosocial: (a) to show love and disagreement; and (b) to start feeling proud when achieving things;

(6) Skills of everyday life: (a) to start using a spoon even if most of the food will be spilled; and (b) to take his sock and shoes off;

(7) Play: (a) to make a tower with 4 blocks; and (b) to start parallel play with other children;

(6) Other: (a) to imitate house works. as well as two out of four words; (b) to develop further joined attention; and (c) the targets were given to parents for work at home.

After a year's intervention the second EDALFA was completed at the age of 47 months (see Figure 3).

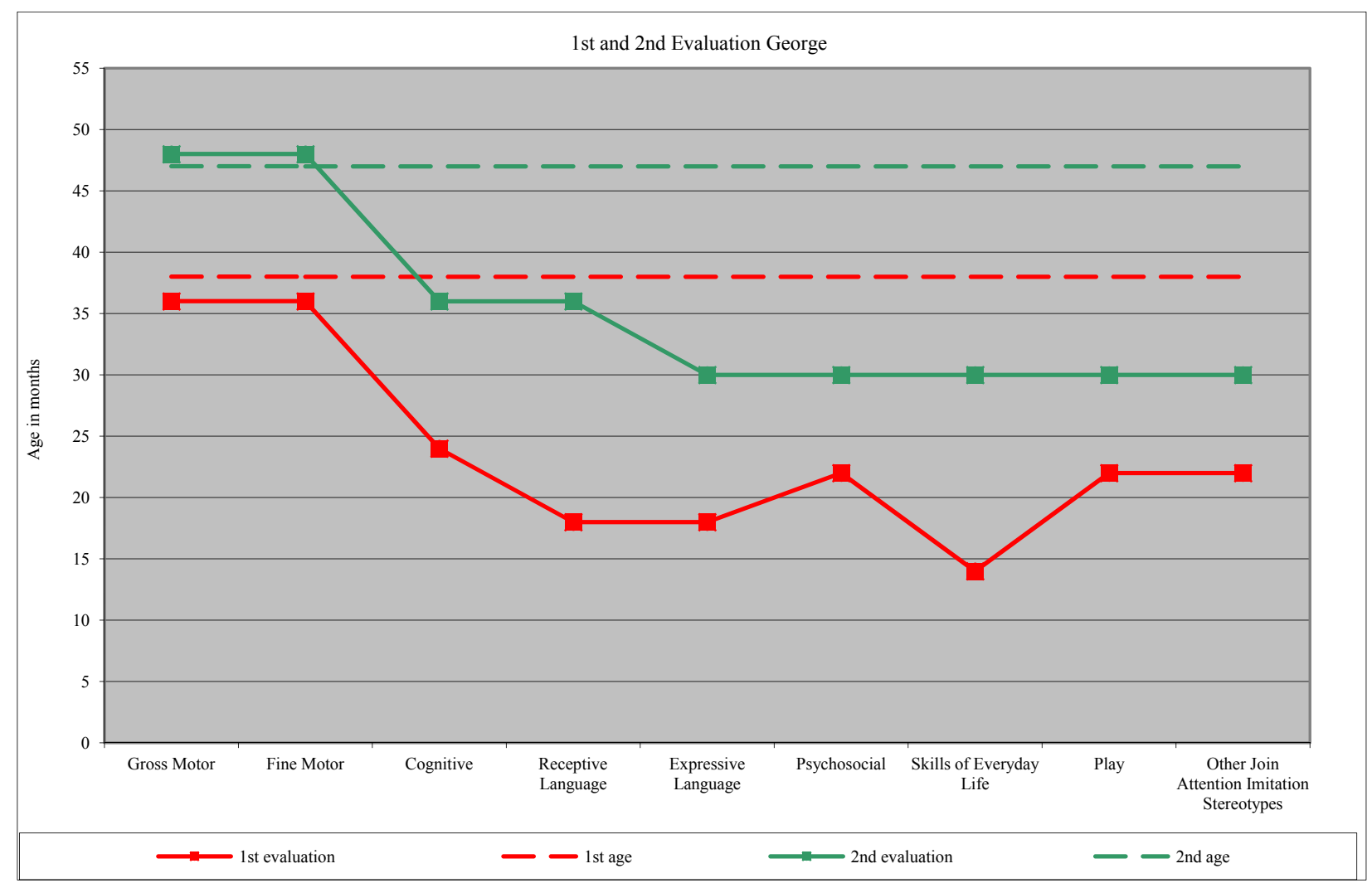

Figure 3. Initial and re-evaluation of the child.

At 47 months, his Gross and fine motion were within the wider typical development range, his cognitive skills at 36 months, his comprehension of speech at 36 months, his expression of speech at 24 months, his psychokinetic skills at 24-30 months, his everyday skills at 24-30 months, his playing at 24-30 and other skills (joined attention, imitation) at 24 -30months.

New targets were made.

\section{Reliability of EDALFA and Usefulness in Clinical Practice}

To examine the reliability of EDALFA, and it is usefulness in clinical practice two studies were conducted 
that compared the EDALFA profiles of children with ASD with those of the Vineland questionnaires answered by the parents.

\section{Study 1}

The sample consisted of thirty children (30), 24 boys and 6 girls (2, 0 yrs to 5 yrs 11 months. mean: 3 yrs 9 months.) diagnosed with ASD (DSM-IV) and accepted at the Day Centre for therapy. For each one of them the Vineland questionnaire was answered by the parents and EDALFA was completed by the treatment team. The following seven measures were common to both tests. Gross and fine motor, communication receptive, and expressive, psychosocial, every day skills, play. A correlation analysis was made between measures shared by the two assessment tools (see Table 4).

Table 4

Correlation Between EDALFA and Vineland: Initial Evaluation: 30 Subjects

\begin{tabular}{|c|c|c|c|c|c|c|c|}
\hline \multirow[b]{2}{*}{ EDALFA } & \multicolumn{7}{|l|}{ Vineland } \\
\hline & Gross motor & Fine motor & Commum. recept & Commun. expr. & Psychosocial & $\begin{array}{l}\text { Everyday } \\
\text { skills }\end{array}$ & Play \\
\hline \multicolumn{8}{|l|}{ Gross motor } \\
\hline \multicolumn{8}{|l|}{ Fine motor } \\
\hline Commum. recept & & & $0.938^{* *}$ & & & & \\
\hline Commun. expr. & & & & $0.935^{* *}$ & & & \\
\hline Psychosocial & & & & & $0.885^{* *}$ & & \\
\hline Everyday skills & & & & & & $0.947^{* *}$ & \\
\hline Play & & & & & & & $0.896^{* *}$ \\
\hline
\end{tabular}

Table 5

Correlation Between EDALFA and Vineland: Follow-up Evaluation: Subjects 15

\begin{tabular}{|c|c|c|c|c|c|c|c|}
\hline \multirow[b]{2}{*}{ EDALFA } & \multicolumn{7}{|l|}{ Vineland } \\
\hline & Gross motor & Fine motor & Commum. recept & Commun. expr. & Psychosocial & $\begin{array}{l}\text { Everyday } \\
\text { skills }\end{array}$ & Play \\
\hline \multicolumn{8}{|l|}{ Gross motor } \\
\hline \multicolumn{8}{|l|}{ Fine motor } \\
\hline Commum. recept & & & $0.938^{* *}$ & & & & \\
\hline Commun. expr. & & & & $0.935^{* *}$ & & & \\
\hline Psychosocial & & & & & $0.885^{* *}$ & & \\
\hline Everyday skills & & & & & & $0.947^{* *}$ & \\
\hline Play & & & & & & & $0.896^{* *}$ \\
\hline
\end{tabular}

\section{Study 2}

Sample: 15 children of the 30 children of the first study were reassessed after completion of a treatment period, and Vineland questionnaires were filled again by the parents. The 15 children were those who remained in therapy for a period necessary for EDALFA re-assessment. To assess progress changes related to treatment a paired $t$-test was carried out between the EDALFA first assessment and re-assessment, as well as between the Vineland first and second questionnaires filled by the parents at the beginning and after a particular therapy period (see Tables 6 and 7). Furthermore, a correlation analysis was also carried out between the reassessments of the two tools EDALFA and Vineland (see Table 5). 


\section{Results}

The correlations between measures of EDALFA and Vineland, on 30 children, were statistically significant (Pearson's $r$ ranging from 0.474 to 0.957 ) (see Table 4) in all corresponding variables of the two measures. Similarly, the correlation between EDALFA and VINELAND on the reassessment of the 15 children were statistically significant (Pearson's $r 0.885$ to 0.947 ) on all 5 common measures (see Table 5). The observed changes on EDALFA and Vineland measures between initial and reassessment values, following a period of therapy, as assessed with the $t$-test were, with one exception, all highly significant (see Tables 6 and 7).

Table 6

T-test EDALFA Between First Evaluation and Follow-up Evaluation

\begin{tabular}{|c|c|c|c|c|c|c|c|}
\hline \multirow{2}{*}{$\begin{array}{l}\text { EDALFA } \\
\text { first evaluation }\end{array}$} & \multicolumn{7}{|c|}{ EDALFA follow-up evaluation } \\
\hline & Gross motor & Fine motor & Commum. recept & Commun. expr. & Psychosocial & Everyday skills & Play \\
\hline Gross motor & $p=0.096$ & & & & & & \\
\hline Fine motor & & $p=0.004$ & & & & & \\
\hline Commum. recept & & & $p=0.000$ & & & & \\
\hline Commun. expr. & & & & $p=0.000$ & & & \\
\hline Psychosocial & & & & & $p=0.001$ & & \\
\hline Everyday skills & & & & & & $p=0.000$ & \\
\hline Play & & & & & & & $p=0.000$ \\
\hline
\end{tabular}

Table 7

T-test VINELAND Between First Evaluation and Follow-up Evaluation

\begin{tabular}{|c|c|c|c|c|c|c|c|}
\hline Vineland & Vineland ree & aluation & & & & & \\
\hline First evaluation & Gross motor & Fine motor & Commum. recept & Commun. expr. & Psychosocial & Everyday skills & Play \\
\hline \multicolumn{8}{|l|}{ Gross motor } \\
\hline \multicolumn{8}{|l|}{ Fine motor } \\
\hline Commum. recept & & & $p=0.000$ & & & & \\
\hline Commun. expr. & & & & $p=0.000$ & & & \\
\hline Psychosocial & & & & & $p=0.000$ & & \\
\hline Everyday skills & & & & & & $p=0.000$ & \\
\hline Play & & & & & & & $p=0.000$ \\
\hline
\end{tabular}

\section{Discussion}

Objective of the present study was the presentation of a tool (EDALFA), developed by a team of therapists, for the clinical appraisal of the child with ASD on a number of variables. The clinical profile of the child and the detailed scale of deficits on each variable provide the steps the therapists will follow in a coordinated effort in the treatment. The profile provided by the scale addresses the problem of clinical variability observed among children with ASD particularly at the child's preschool level. A variety of difficulties, e.g., attention, feeding, speech, etc., could not be dealt with by one professional discipline. The need for multidisciplinary involvement is necessary. The therapeutic goals may also be shared with parents and teachers involved. The absence of common therapy goals may make the therapeutic effort chaotic.

No doubt the clinical practice may lead to refinement and improvement of the steps which provide the 
hierarchical targets by EDALFA that should be addressed. Essential part of EDALFA is the developmental scale of the typically developing child which offers norms and skills that the typical child at a particular age level is supposed to have mastered. These steps serve as mini milestone for the therapists to use and compare the child's development level. This tool has been in use successfully at the Day Centre for Children with ASD for seven years now with minimal adjustments.

The comparison between the scores of EDALFA, and Vineland answered by the parents on measures with similar content in two different times, first with 30 and second on follow up with 15 subjects showed highly significant correlations. These associations indicate that EDALFA reliably assesses characteristics measured by Vineland.

\section{Limitations and Recommendations}

This paper is a pilot study. Further research with larger samples is needed.

\section{References}

Arvedson, J., \& Brodsky, L. (2002). Pediatric swallowing and feeding: Assessment and management (2nd ed.). Albany, N.Y.: Singular Thomson Learning.

Arvedson, J. C. (2007). Pediatric dysphasia: Decision making with complex patients. Notes of Athens Seminar on Dysphagia, 2010.

Ayres, A. J. (2005). Sensory integration and the child. Torrance, C.A.: Western Psychological Services.

Bzoch, K. R., \& League, R. (2000). Receptive expressive emergent language test (REEL-3). Austin, M.N.: Pro-Ed.

Dawson, G., Rogers, S., Munson, J., Smith, M. et al. (2010). Randomized, controlled trial of an intervention for toddlers with autism: The early start denver model. Pediatrics, 125(1), 17-23.

Dziuk, M. A., Gidley, J. K., Apostu, A., Mahone, E. M., Denkia, M. B., \& Mostofski, S. H. (2007). Dyspraxia in autism: Association with motor, social, and communicative deficits. Developmental Medicine and Child Neurology, 49(10), $734-739$.

Emond, A., Emmett, P., Steer, C., \& Golding, J. (2010). Feeding symptoms, dietary patterns, and Ggowth in young children with autism spectrum disorders. Pediatrics, 126(2), 337-342.

Fewell, R. R., \& Folio, M. R. (2000). Peabody developmental motor scales (2nd ed.). Austin: Pro Ed.

Georgiades, S., Szatmari, P., Zwaigenbaum, L., Bryson, S., \& Brian, J. (2013). A prospective study of autistic-like traits in unaffected siblings of probands with autism spectrum disorder. JAMA Psychiatry, 70, 42-48.

Helt, M., Kelley, E., Kinsbourne, M., Pandey, J., Boorstein, H., Herbert, M. ...Fein, D. (2008). Can children with autism recover? If so, how? Neuropsychology Review Dec., 18(4), 339-366.

Hindley, C. B. (1960). The griffiths scale of infant development scores and predictions from 3 to 18 months. Journal of Child Psychology and Psychiatry, 1, 99-112.

Kotsopoulou, A., Gasteratos, A., Georgiou, A., Gerantoni, S., Gyftoyianni, M., Sakellari, M., ... \& Hasaki, A. (2009). EDALFA: A multidisciplinary team's tool for the assessment of the functional level of children with ASD. Messolonghi, Greece: EPSYPEA.

Kotsopoulou, A., Gasteratos, A., Georgiou, A., Gerantoni, S., Gyftoyianni, M., Sakellari, M., ... \& Hasaki, A. (2010). A tool of the Interdisciplinary team for the assessment of the level of functioning of children with autism spectrum disorders (EDALFA). Oral presentation on the 28th World Congress of the International Association of Logopedics and Phoniatrics (IALP), Athens Greece.

Mecham, J. (1958). Verbal language development scale. In V. P. Roman (1980), The relationship between language ages of preschool children derived from a parent informant scale and language ages derived from tests administered directly to the preschool child. Language, Speech, and Hearing Services in Schools, 11, 50-55.

Ozonoff, S., Iosif, A. M., Baguio, F., \& Hepburn, S. (2010). A prospective study of the emergence of early behavioral signs of autism. J. Amer Acad Child \& Adoles Psychiatry, 49, 256-266.

Schreck, K. A., Williams, K., \& Smith, A. F. (2004). A comparison of eating behaviors between children with and without autism. Journal of Autism and Developmental Disorders, 34(4), 433-438.

Scott, D. T., \& Winnie, D. (2007). Sensory processing in children with and without autism: A comparative study using the short sensory profile. American Journal of Occupational Therapy, 61(2), 190-200. 
Sparrow, S., Cicchetti, D., \& Balla, D. A. (2005). Vineland adaptive behavior Scales: Survey form (2nd ed.). Circle Pines, M.N.: American Guidance Service.

Sullivan, M., Finelli, J., Marvin, A., Garrett-Mayer, E., Bauman, M., \& Landa, R. (2007). Response to joint attention in toddlers at risk for autism spectrum disorder: A prospective study. J. Autism Dev Disord, 37(1), 37-48.

Tierney, C. D., Kurtz, M., \& Souders, H. (2012). Clear as mud: Another look at autism, childhood apraxia of speech and auditory processing. Current Opinion of Pediatrics, 24(3), 394-399.

Watson, L., Baranek, G., Crais, E., Reznick, S. J., Dykstra, J., \& Perryman, T. (2007). The first year inventory: Retrospective parent response to a questionnaire designed to identify one-year-olds at risk for autism. J. Autism Dev Disord, 37, 49-61.

Zimmerman, I. L., Steiner, V. G., \& Pond, R. E. (1992). Preschool language scale-3 (PLS-3). San Antonio, T.X.: The Psychological Corporation. 